\title{
G-Series Nerve Agent GF
}

National Cancer Institute

\section{Source}

National Cancer Institute. G-Series Nerve Agent GF. NCI Thesaurus. Code C161530.

A man-made highly toxic org anic phosphate that is more persistent and less lethal than its nerve agent predecessor, Sarin. It is a colorless liquid with a sweet, musty odor that is rapidly absorbed through skin and inhibits acetylcholinesterase, increasing the neurotransmitter acetylcholine at central and peripheral neuronal synapses. 